\title{
Carotid body and cardiorespiratory alterations in intermittent hypoxia: the oxidative link
}

\author{
R. Del Rio, E.A. Moya and R. Iturriaga
}

ABSTRACT: Intermittent hypoxia, a feature of obstructive sleep apnoea, potentiates ventilatory hypoxic responses, alters heart rate variability and produces hypertension, partially owing to an enhanced carotid body responsiveness to hypoxia. Since oxidative stress is a potential mediator of both chemosensory and cardiorespiratory alterations, we hypothesised that an antioxidant treatment may prevent these alterations.

Accordingly, we studied the effects of ascorbic acid (1.25 $\mathrm{g} \cdot \mathrm{L}^{-1}$ drinking water) on plasma lipid peroxidation, nitrotyrosine and inducible nitric oxide synthase (iNOS) immunoreactivity in the carotid body, ventilatory and carotid chemosensory responses to acute hypoxia, heart rate variability and arterial blood pressure in male Sprague-Dawley rats exposed to $5 \% \mathrm{O}_{2}$; 12 episodes $\cdot h^{-1} ; 8 \mathrm{~h} \cdot \mathrm{day}^{-1}$ or sham condition for 21 days.

Intermittent hypoxia increased plasma lipid peroxidation, nitrotyrosine and iNOS expression in the carotid body, enhanced carotid chemosensory and ventilatory hypoxic responses, modified heart rate variability and produced hypertension. Ascorbic acid prevented the increased plasma lipid peroxidation and nitrotyrosine formation within the carotid body, and the enhanced carotid chemosensory and ventilatory responses to hypoxia, as well as heart rate variability alterations and hypertension.

The present results support an essential role for oxidative stress in the generation of carotid body chemosensory potentiation and systemic cardiorespiratory alterations induced by intermittent hypoxia.

\section{KEYWORDS: Ascorbic acid, carotid body, intermittent hypoxia, sleep apnoea}

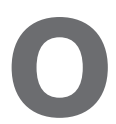

bstructive sleep apnoea (OSA), a highly prevalent sleep-breathing disorder is recognised as an independent risk factor for hypertension [1, 2]. OSA, characterised by recurrent episodes of partial or complete upper airway obstruction during sleep, produces chronic intermittent hypoxia $(\mathrm{CIH})$, which is considered the main factor for hypertension [2,3]. Sympathetic activation, oxidative stress and inflammation have been proposed as potential mechanisms for OSA-induced hypertension [3-5]. However, conclusive evidence from studies in OSA patients has been difficult to establish because of concomitant comorbidities [3]. Thus, animal CIH models simulating hypoxic reoxygenation cycles are used to study OSA pathological mechanisms. Similarly to that which is observed in OSA patients, $\mathrm{CIH}$ exposed animals show enhanced sympathetic discharges in response to hypoxia, alterations of heart rate variability (HRV) and develop systemic hypertension [6, 7].

It has been proposed that a major contributing mechanism to $\mathrm{CIH}$-induced sympathetic activation is the potentiation of the carotid body (CB) chemoreflex response to hypoxia [5, 7-11]. Indeed, recently diagnosed OSA patients show enhanced ventilatory and cardiovascular hypoxic responses attributed to a CB chemosensory potentiation $[9,10]$. Moreover, studies performed in animal models provided evidence that $\mathrm{CIH}$ enhances $\mathrm{CB}$ chemosensory and ventilatory responses to hypoxia and produces long-term facilitation of respiratory motor activity $[12,13]$. The mechanisms underlying the $\mathrm{CIH}$-induced $\mathrm{CB}$ chemosensory potentiation are not well known. The enhanced $C B$ hypoxic reactivity has been attributed to increased levels of endothelin-1 [14] and free radicals in the

\section{AFFILIATIONS}

Laboratorio de Neurobiología, Facultad de Ciencias Biológicas, Pontificia Universidad Católica de Chile, Santiago, Chile.

\section{CORRESPONDENCE}

R. Iturriaga

Laboratorio de Neurobiología

Facultad de Ciencias Biológicas

Pontificia Universidad Católica de

Chile

Casilla 114-D

Santiago

Chile

E-mail: riturriaga@bio.puc.cl

Received:

Oct 062009

Accepted after revision:

Nov 182009

First published online:

Dec 082009 
CB $[12,15]$, but nitric oxide (NO) and pro-inflammatory molecules may also be involved [8]. Since reactive oxygen (ROS) and nitrogen species are potential mediators of cardiovascular alterations in OSA patients [3-5] and CIH-exposed animals [15-17], we hypothesised that an antioxidant treatment may prevent both $\mathrm{CB}$ and cardiorespiratory altered functions. Thus, we studied the effects of ascorbic acid on the potentiation of CB chemosensory and ventilatory hypoxic responses, HRV alterations and the hypertension induced by $\mathrm{CIH}$ in rats. Malondialdehyde (MDA) assay was used as a systemic oxidative stress marker, along with 3nitrotyrosine (3-NT) and inducible nitric oxide synthase (iNOS) immunoreactivity as oxidative stress markers in the CB. This study was performed in anaesthetised rats, a necessary condition to record $\mathrm{CB}$ chemosensory neural activity.

\section{METHODS}

\section{Animals and intermittent hypoxic exposure}

Experiments were performed on male Sprague-Dawley rats (200 g), fed with standard diet ad libitum and kept on a 12-h light/dark schedule (08:00-20:00 h). The protocol was approved by the Bioethical Committee of the Biological Sciences Faculty, P. Universidad Católica de Chile, Santiago, Chile. Unrestrained, freely moving rats housed in individual chambers $(12 \mathrm{~cm} \times 35 \mathrm{~cm}, 2.2 \mathrm{~L})$ were exposed to $5 \%$ inspired $\mathrm{O}_{2}$ for $20 \mathrm{~s}$ followed by room air for $280 \mathrm{~s} ; 12$ episodes $\cdot \mathrm{h}^{-1}$; $8 \mathrm{~h} \cdot$ day $^{-1}$ for 21 days. The hypoxic pattern was applied from 08:00 to 16:00 h. A computerised system based on solenoid valves controls the alternating cycles of $\mathrm{N}_{2}$ and room air [7]. In the sham condition, the $\mathrm{N}_{2}$ gas was replaced by means of flushing an equal flow of compressed air into chambers. Room temperature was kept at $23-25^{\circ} \mathrm{C}$. Ascorbic acid $\left(1.25 \mathrm{~g} \cdot \mathrm{L}^{-1}\right)$ was administered through the drinking tap water from the first day of $\mathrm{CIH}$ exposure. The water solution was freshly prepared every day, and preserved in dark containers to avoid oxidation. Rats were randomly assigned into experimental groups. Ascorbic acid was administered to rats exposed to $\mathrm{CIH}$ (CIH-AA) or sham condition (sham-AA). Fluid intake was not significantly different between all groups $\left(\sim 10 \mathrm{~mL} \cdot\right.$ day $\left.^{-1}\right)$.

\section{Physiological recordings}

Recordings were performed $\sim 16 \mathrm{~h}$ after the end of the last hypoxic cycle. Rats were anaesthetised with sodium pentobarbitone $\left(40 \mathrm{mg} \cdot \mathrm{kg}^{-1}\right.$; intraperitoneal) followed by additional doses when necessary to maintain a level of surgical anaesthesia, placed in supine position and the rectal temperature was maintained at $38.0 \pm 0.5^{\circ} \mathrm{C}$ with a regulated heating pad. The trachea was cannulated for airflow recording, and connected to a pneumotachograph to measure tidal volume $(V \mathrm{~T})$, respiratory frequency, and minute inspiratory volume $\left(V^{\prime} \mathrm{I}\right)$. One carotid artery was cannulated with a P50-polyethylene tube, filled with $50 \mathrm{IU} \cdot \mathrm{mL}^{-1}$ of heparin solution for measuring arterial blood pressure (BP) with a transducer (Statham P23; Statham Instruments, Inc., Oxnard, CA, USA). However, in experiments designed to record CB chemosensory discharges and ventilatory responses, the carotid artery was not cannulated, in order to avoid any interference with the blood supply of the $\mathrm{CB}$ region. Heart rate was obtained from the ECG signal recorded using the II lead, and the mean arterial blood pressure (MABP) from the BP signal. Signals were acquired with an analogue-digital system PowerLAB 8SP (ADInstruments Pty Ltd, Castle Hill, Australia), calibrated and analysed with the Chart 6.1-Pro software (ADInstruments Pty Ltd). To assess the contribution of the peripheral chemoreceptors on the $\mathrm{CIH}$-induced changes in the ventilatory reflex, we measured ventilatory responses elicited by isocapnic levels of oxygen tension $\left(\mathrm{PO}_{2}\right)(5-670 \mathrm{mmHg})$, maintained until the response was in a semi-steady state $(\sim 10-20 \mathrm{~s})$.

\section{Heart rate variability}

The ECG was recorded prior to any manoeuvre for $10 \mathrm{~min}$ at $2 \mathrm{kHz}$. After the acquisition, the signal was explored for detection of ectopic QRS complexes and R-R intervals were measured. The HRV was analysed with the HRV module of the Chart 6.1-Pro software. The power spectrum of R-R interval data was obtained using a Fast Fourier Transform algorithm after application of the Hann window. The spectrum of R-R intervals was assessed using the following frequency bands: very-low frequency: DC-0.04 Hz, low frequency (LF): $0.04-$ $0.6 \mathrm{~Hz}$ and high frequency (HF): $0.6-2.4 \mathrm{~Hz}$ [18]. Calculations considered the relative power of the LF and HF powers expressed as normalised units and the LF/HF ratio.

\section{CB chemosensory recordings}

The CB chemosensory discharge was measured as previously described [11]. Briefly, one carotid sinus nerve was dissected and placed on a pair of Pt electrodes and covered with warm mineral oil. The neural signal was pre-amplified (Grass P511; Astro-Med Inc., West Warwick, RI, USA), filtered (30-500 Hz) and fed to an electronic spike-amplitude discriminator allowing the selection of action potentials of given amplitude above the noise. Selected action potentials were counted with a frequency meter to assess the $C B$ chemosensory frequency of discharge $(f x)$, expressed in $\mathrm{Hz}$. In the $\mathrm{CB}$ recordings, the contralateral carotid sinus nerve was cut to prevent vascular and ventilatory effects caused by the activation of chemosensory reflexes (see online supplementary data). The chemosensory discharge was measured at several isocapnic levels of $\mathrm{PO}_{2}$ (5-670 $\mathrm{mmHg})$, maintained until the response was in semisteady state $(\sim 10-20 \mathrm{~s})$. Rats breathed spontaneously during the whole experiment. At the end of the experiments, rats were killed by an overdose of pentobarbitone $\left(100 \mathrm{mg} \cdot \mathrm{kg}^{-1}\right.$ i.p. $)$.

\section{Lipid peroxidation assay}

A spectrophotometric assay for detection of thiobarbituric acid reactive substances (TBARS) was used as an oxidative stress marker. Blood samples were collected through the carotid artery, and placed in heparinised ice-cold microcentrifuge tubes. Plasma was separated by centrifugation and stored at $-80^{\circ} \mathrm{C}$. MDA was used as a standard, and the level of TBARS was reported as $\mathrm{nmol} \cdot \mathrm{mL}^{-1}$ of MDA.

\section{Immunohistochemical detection of 3-NT and iNOS in the $C B$}

Anaesthetised rats were perfused intracardially with PBS at pH 7.4 for 10 min followed by buffered $4 \%$ paraformaldehyde (Sigma, St Louis, MO, USA). Carotid bifurcations containing the $\mathrm{CB}$ were dissected and post-fixed in the same fixative solution, dehydrated in ethanol, included in paraffin, cut in $5 \mu \mathrm{m}$ sections and mounted on silanised slides. Deparaffinised samples were incubated with $\mathrm{H}_{2} \mathrm{O}_{2}$ to inhibit endogenous peroxidase and then in blocking serum solution (Vector Laboratories Inc., Burlingame, CA, USA). The slices were 
incubated with an anti-3-NT polyclonal antibody (1:20 in PBS/ bovine serum albumin 1\%; Molecular Probes A-21285) or an anti-iNOS polyclonal antibody (1:200 in PBS/bovine serum albumin 1\%; Sigma no. 7782; Sigma) overnight at $4^{\circ} \mathrm{C}$. Slices were incubated with an universal biotinylated secondary antibody followed by a ready-to-use stabilised $\mathrm{ABC}$ reagent (Vectastain Elite ABC Kit; Vector Laboratories Inc.), and revealed with 3,3-diaminobenzidine tetrachloride (Sigma). Samples were counterstained with Harris' haematoxylin and mounted. Photomicrographs were taken at $100 \times$ with a CCD camera coupled to an Olympus CX 31 microscope (Olympus Corporation, Tokyo, Japan), digitised and analysed with the ImageJ software (National Institutes of Health, Bethesda, MD, USA). The immunoreactive intensity, averaged from eight fields for each sampled CB, was expressed as optical integrated intensity.

\section{Statistical data analysis}

Data were expressed as mean \pm SEM. Differences between three or more groups were assessed with one or two-way ANOVA tests, followed by Newman-Keuls post hoc comparisons.

\section{RESULTS}

The effects of $\mathrm{CIH}$ on mean $\mathrm{BP}$, heart rate, $V^{\prime} \mathrm{I}, V \mathrm{~T}$, respiratory rate and basal $f x$ measured at the beginning of the recordings while rats breathed spontaneously room air are summarised in table 1. Mean BP was higher in the $\mathrm{CIH}$-treated rats than in the other groups $(\mathrm{p} \leqslant 0.05)$, but heart rate did not differ between any groups. Basal $V \mathrm{~T}, V^{\prime} \mathrm{I}$ and respiratory rate in $\mathrm{CIH}$-treated rats were not significantly different from those recorded from sham, CIH-AA or sham AA-rats. However, basal $f x$ was significantly higher $(\mathrm{p} \leqslant 0.05)$ in rats exposed to $\mathrm{CIH}$ $(80.7 \pm 11.4 \mathrm{~Hz} ; \mathrm{n}=11)$ than basal $f x$ measured in sham rats $(47.8 \pm 5.6 \mathrm{~Hz} ; \mathrm{n}=8)$. Treatment with ascorbic acid prevented the increase of basal $f x$ in rats exposed to $\mathrm{CIH}$.

Rats exposed to $\mathrm{CIH}$ showed higher ventilatory responses as compared with sham, CIH-AA and sham-AA rats (fig. 1; $p \leqslant 0.001$, two-way ANOVA). The CB chemosensory response to acute hypoxia was also enhanced by $\mathrm{CIH}$ (figs $1 \mathrm{c}$ and 2). The two-way ANOVA analysis indicated that the overall $\mathrm{CB}$ chemosensory curve for $\mathrm{PO}_{2}$ was different in $\mathrm{CIH}$ rats $(\mathrm{p} \leqslant 0.001)$ compared with the other groups. The post hoc test indicates that $\mathrm{CB}$ chemosensory discharges were higher $(p \leqslant 0.01)$ not only in the hypoxic range, but also in normoxia. The ascorbic acid treatment prevented the enhanced ventilatory and $\mathrm{CB}$ chemosensory responses induced by $\mathrm{CIH}$.

As is shown in table 1, CIH produced a significant increase of MABP, while ascorbic acid treatment prevented the increase in $\mathrm{MABP}$ in $\mathrm{CIH}$ rats. In addition, $\mathrm{CIH}$ produced a marked modification of the distribution of the relative spectrum power of LF and HF frequency bands, which was prevented by ascorbic acid (figs 3 and 4). Figure 4 shows the summary of $\mathrm{CIH}$ effects on LF/HF ratio, LF and HF bands. Rats exposed to $\mathrm{CIH}$ had a significantly higher $\mathrm{LF} / \mathrm{HF}$ ratio $(0.56 \pm 0.08)$ than the other groups (fig. $4 \mathrm{a}$ ) due to a reduction of the HF band and a concomitant increase of the relative power of the LF band (fig. $4 \mathrm{~b}$ and $\mathrm{c}$ ). We found a statistically significant linear correlation $(\mathrm{r} \geqslant 0.45, \mathrm{p}<0.05)$ between the ventilatory response to $5 \%$ inspired $\mathrm{O}_{2}$ and $\mathrm{LF} / \mathrm{HF}$ ratio only in the $\mathrm{CIH}$-treated rats. On the contrary, in the other groups, the correlation coefficients were low and lacked statistical significance (see online supplementary material).

We found a significant increase of 3-NT immunoreactivity (fig. 5) and iNOS immunoreactivity (fig. 6) in the CB from $\mathrm{CIH}$ rats. The 3-NT immunoreactivity and iNOS immunoreactivity were found in chemoreceptor cell clusters and in the endothelium of interlobular vessels (figs 5 and 6). The relative expression level of 3-NT immunoreactivity was lower in the CBs from CIH-AA than that of $\mathrm{CIH}$ rats. Negative controls, omitting the primary antibody were consistently devoid of staining (not shown). Figure 7 shows the quantification of the effects of $\mathrm{CIH}$ on 3-NT immunoreactivity (fig. 7a), iNOS immunoreactivity (fig. 7b) and plasma MDA (fig. 7c). The $\mathrm{CIH}$-treated rats showed 2.5-fold increase in plasma MDA compared with sham, CIH-AA and sham-AA-treated rats $(p \leqslant 0.01$, one-way ANOVA). Rats exposed to $\mathrm{CIH}$, but treated with ascorbic acid showed a $70 \%$ of reduction in MDA plasma level compared with the $\mathrm{CIH}$ rats.

\section{DISCUSSION}

The main finding of this study shows that ascorbic acid, which reduced the increased MDA plasma level and the 3-NT in the $\mathrm{CB}$, prevented the potentiation of $\mathrm{CB}$ chemosensory and ventilatory responses to acute hypoxia, as well as HRV

\section{TABLE 1 Effect of chronic intermittent hypoxia $(\mathrm{CH})$ on physiological variables at normoxia}

\begin{tabular}{|c|c|c|c|c|c|c|c|c|}
\hline \multirow[t]{2}{*}{ Variable } & \multicolumn{2}{|c|}{ Sham } & \multicolumn{2}{|l|}{$\mathrm{CIH}$} & \multicolumn{2}{|c|}{ Sham-AA } & \multicolumn{2}{|c|}{ CIH-AA } \\
\hline & Mean \pm SEM & $\mathbf{n}$ & Mean \pm SEM & $\mathbf{n}$ & Mean \pm SEM & $\mathbf{n}$ & Mean \pm SEM & $\mathbf{n}$ \\
\hline MABP $\mathrm{mmHg}$ & $111 \pm 4$ & 15 & $138 \pm 3^{\star \star \star}$ & 16 & $117 \pm 6^{\# \#}$ & 9 & $109 \pm 6^{\# \# \#}$ & 10 \\
\hline fc beats $\min ^{-1}$ & $427 \pm 17$ & 15 & $403 \pm 14$ & 16 & $394 \pm 15$ & 9 & $386 \pm 17$ & 10 \\
\hline$V_{T} \mathbf{m L} \cdot \mathbf{k g}^{-1}$ & $4.4 \pm 0.5$ & 14 & $3.6 \pm 0.4$ & 22 & $3.4 \pm 0.2$ & 11 & $3.6 \pm 0.2$ & 11 \\
\hline fR breaths $\cdot \min ^{-1}$ & $78 \pm 6$ & 14 & $74 \pm 3$ & 22 & $80 \pm 4$ & 11 & $78 \pm 3$ & 11 \\
\hline$V^{\prime} ı \mathrm{~mL} \cdot \mathrm{min}^{-1} \cdot \mathrm{kg}^{-1}$ & $369 \pm 58$ & 14 & $273 \pm 37$ & 22 & $286 \pm 26$ & 11 & $291 \pm 39$ & 11 \\
\hline Basal $f_{x} \mathrm{~Hz}$ & $48 \pm 6$ & 8 & $81 \pm 11^{* *}$ & 11 & $42 \pm 4^{\# \#}$ & 5 & $47 \pm 4^{\# \#}$ & 10 \\
\hline
\end{tabular}



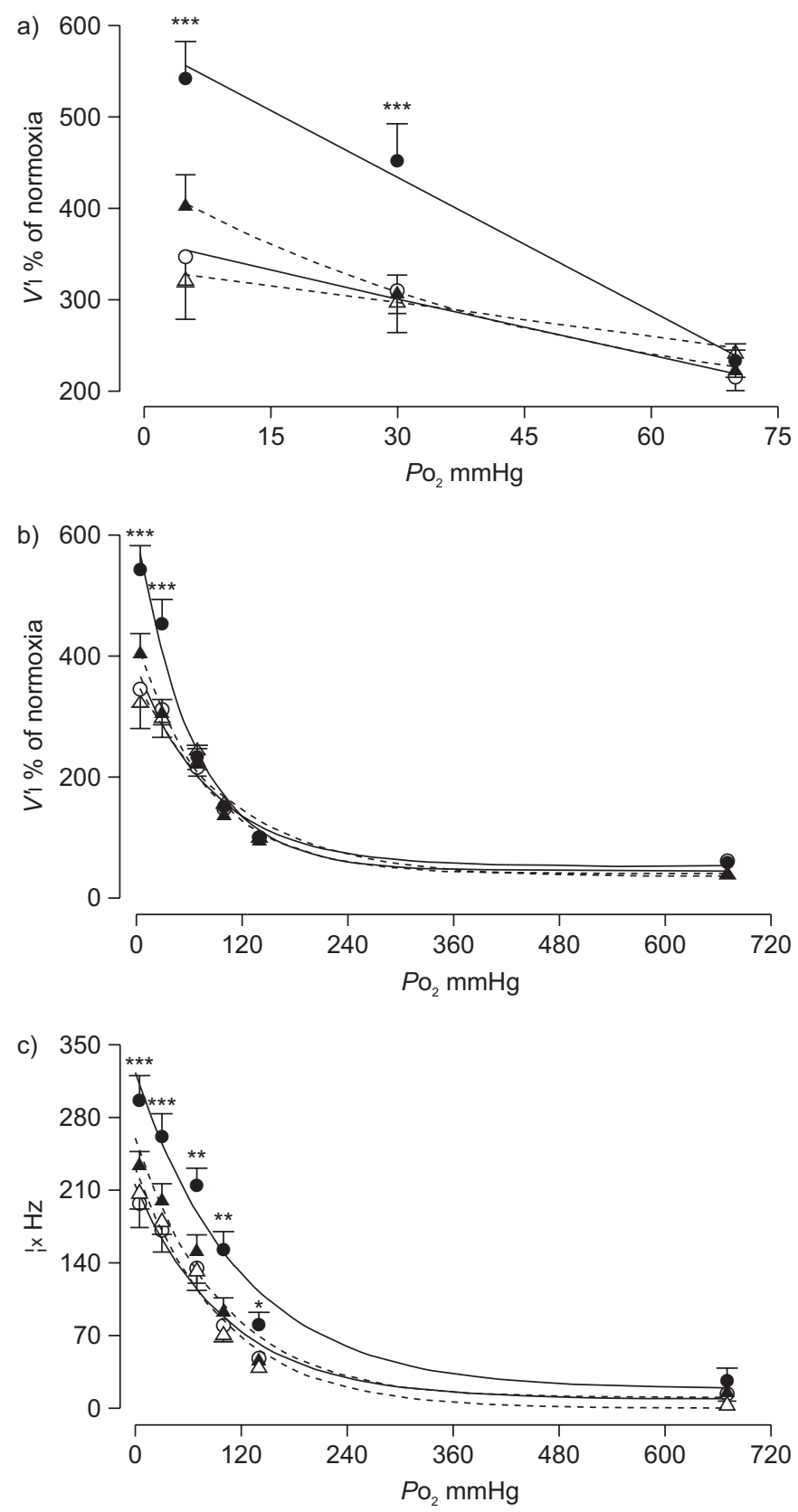

FIGURE 1. Effects of ascorbic acid on ventilatory ( $a$ and $b$ ) and carotid body chemosensory (c) responses to several levels of inspired oxygen tension $\left(\mathrm{PO}_{2}\right)$ in sham rats $(O)$, chronic intermittent hypoxia $(\mathrm{CIH})$ exposed rats $(\bullet)$, sham rats treated with ascorbic acid (sham-AA; $\triangle$ ) and rats exposed to $\mathrm{ClH}$ treated with ascorbic acid (CIH-AA; $\mathbf{\Lambda})$. V'I: ventilatory minute expressed as per cent of normoxia in $22 \mathrm{ClH}$ rats, 20 sham, $11 \mathrm{ClH}$-AA and eight sham-AA rats. $\mathrm{fx}$ : carotid chemosensory frequency of discharge measured in $11 \mathrm{CIH}$ rats, eight sham, 10 $\mathrm{CIH}-\mathrm{AA}$ and four sham-AA rats. ${ }^{* *}: \mathrm{p} \leqslant 0.001 ; * *: \mathrm{p} \leqslant 0.01 ;$ and $*: p \leqslant 0.05$ compared with sham control.

alterations and the hypertension induced by $\mathrm{CIH}$. Thus, the present results support the proposal that oxidative stress plays an essential role in the potentiation of $C B$ chemosensory responses to acute hypoxia, as well as in the cardiorespiratory alterations induced by $\mathrm{CIH}$.
A growing body of evidence supports the idea that an enhanced $\mathrm{CB}$ chemosensory responsiveness contributes to the progression of the cardiorespiratory alterations induced by OSA. FLETCHER et al. [5] were the first to propose that the CB contributes to the hypertension induced by $\mathrm{CIH}$, because they found that bilateral $\mathrm{CB}$ denervation prevented the increased sympathetic discharges and the hypertension in rats exposed to $\mathrm{CIH}$ for 35 days. This proposal had received further support since recordings of $\mathrm{CB}$ chemosensory discharges showed that $\mathrm{CIH}$ augments $\mathrm{CB}$ chemosensory discharges in normoxia and hypoxia $[10,11,14]$, and potentiates ventilatory responses to hypoxia $[11,12,19]$.

The alteration of the autonomic balance resulting from the enhanced hypoxic CB chemosensitivity is thought to be involved in the HRV alterations, the reduction of the efficiency of the baroreflex control of heart rate, and the hypertension in OSA patients [20, 21] and animals exposed to CIH [11, 18, 22]. The present results confirmed our previous observation that cyclic hypoxic stimulation of the $\mathrm{CB}$ induces selective alterations of HRV [11, 22]. LF and HF bands are related to autonomic control on the heart rate. While the HF band has been associated mainly to cardiac parasympathetic efferent activity, the LF band is believed to be modulated mostly by the sympathetic tone [21]. Recently, LAI et al. [18] found that the LF/HF ratio measured from BP was significantly higher in $\mathrm{CIH}$-exposed conscious rats, related to control rats kept under normoxic conditions. They found a significant increase of LF/ HF started 5 days after onset of the CIH exposure that lasted for the 30 days of the experiment. The elevation of LF/HF ratio was due to an increase of the LF band, without significant changes of HF. Similar to these findings, the present data show that $\mathrm{CIH}$ increased LF/HF mainly due to an increased power of the LF band, but we also found a significant reduction of the $\mathrm{HF}$ power, suggesting that $\mathrm{CIH}$ modified the autonomic balance of heart rate, with a relative predominance of the sympathetic over parasympathetic activity. Moreover, we found that $\mathrm{CIH}$-induced increase in the LF/HF ratio significantly correlates with the enhanced ventilatory response to acute hypoxia (see online supplementary data). Patients with long or recently diagnosed OSA show an increased LF/HF ratio, with a relative predominance of the LF band and a reduced contribution of the HF band [20]. Thus, rats exposed to $\mathrm{CIH}$ in this study, similar to OSA patients, show an increased LF/HF ratio suggesting the existence of changes in the autonomic control of heart rate.

Some methodological issues are needed to discuss. We used a $\mathrm{CIH}$ pattern consisting of 12 episodes $\cdot \mathrm{h}^{-1}\left(5 \% \mathrm{O}_{2}\right.$ for $\left.20 \mathrm{~s}\right)$ instead of the protocol of 30 episodes $\cdot \mathrm{h}^{-1}\left(10 \% \mathrm{O}_{2}\right.$ for $\left.90 \mathrm{~s}\right)$ utilised in rodents [19], because it is well established that the former pattern enhances the $\mathrm{CB}$ chemosensory response to acute hypoxia in rats [11] and cats [20]. Although it is possible that the 30 episodes $\cdot h^{-1}$ pattern may evoke different results, it is noteworthy that it also enhances the ventilatory responses to hypoxia in rats [19], suggesting that the $\mathrm{CB}$ chemoreflex responses to hypoxia is potentiated. One limitation of the present study is the use of anaesthesia necessary to record CB chemosensory discharges. Since anaesthesia may depress cardiorespiratory reflexes and sympathetic outflow [23], we cannot preclude a depressor effect of pentobarbitone on ventilatory and cardiovascular responses. 

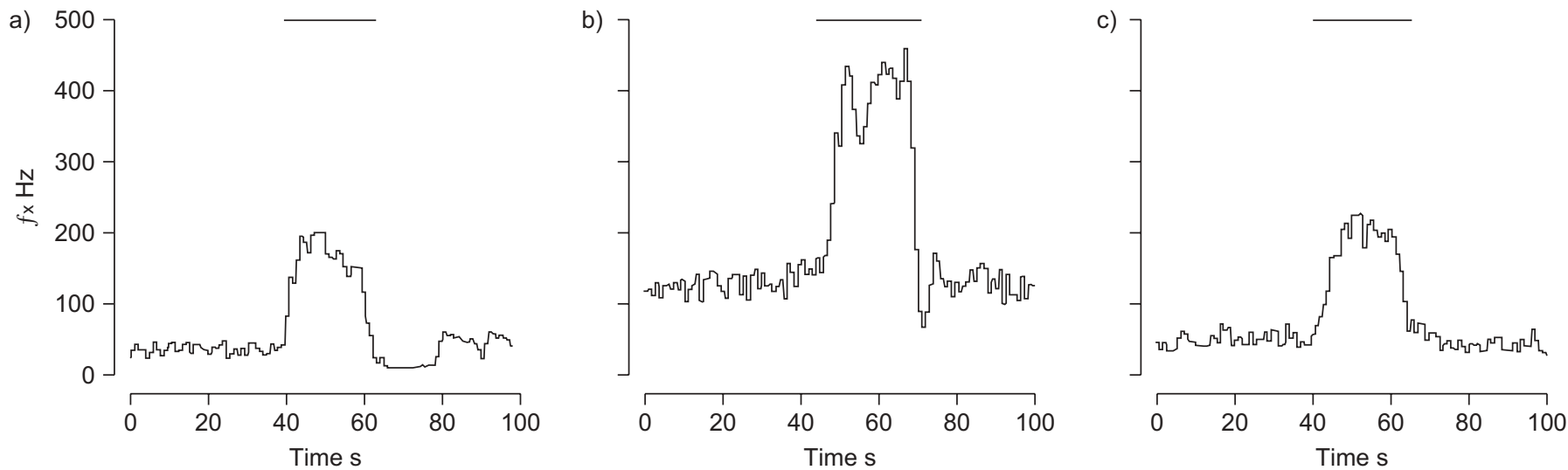

FIGURE 2. Representative recordings of carotid body chemosensory responses to brief hypoxic stimulus (bars indicate inspired oxygen tension $\sim 5 \mathrm{mmHg}$ ) in a) one sham-treated, b) one chronic intermittent hypoxia $(\mathrm{ClH})$-treated rat and c) one rat exposed to $\mathrm{ClH}$ treated with ascorbic acid. $f x$ : carotid chemosensory frequency of discharge.

Nonetheless, clinical assessment of the depth of anaesthesia did not show apparent differences among the experimental groups.

PENG and co-workers [12, 15] found evidence of the involvement of ROS in the potentiation of CB chemosensory responses to hypoxia induced by $\mathrm{CIH}$. They reported that pretreatment of rats for 10 days before $\mathrm{CIH}$ exposure with the superoxide dismutase mimetic manganese(III) tetrakis (1-methyl-4-pyridyl) porphyrin pentachloride (MnTMPyP) prevents the $\mathrm{CB}$
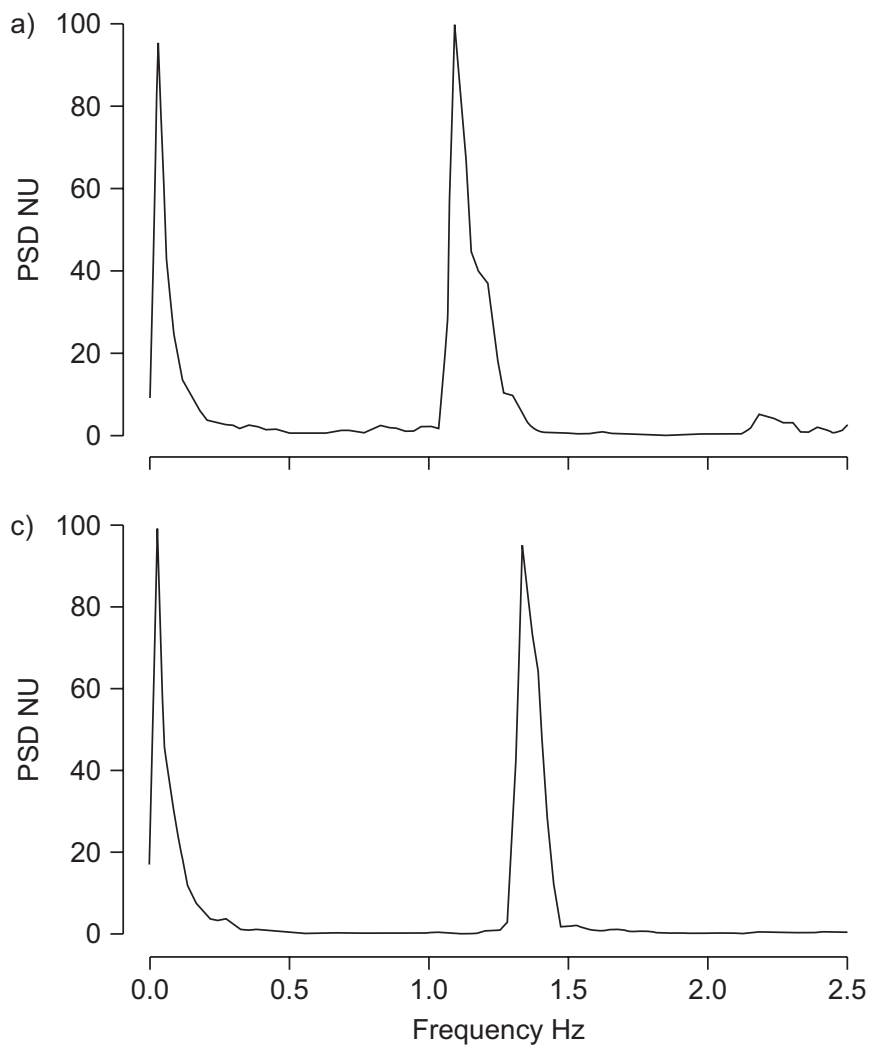

chemosensory facilitation induced by repetitive hypoxia in CBs from rats exposed to $\mathrm{CIH}$. However, when MnTMPyP was applied $15 \mathrm{~min}$ after the onset of the chemosensory facilitation, it failed to block the following increased chemosensory discharges. On the contrary, catalase (an $\mathrm{H}_{2} \mathrm{O}_{2}$ scavenger) applied $15 \mathrm{~min}$ after the onset of the chemosensory facilitation blocked the increased chemosensory discharges. These observations suggest that $\mathrm{O}_{2} \cdot-$ and $\mathrm{H}_{2} \mathrm{O}_{2}$ may contribute to the $\mathrm{CB}$ chemosensory potentiation induced by $\mathrm{CIH}$, but it is not b)

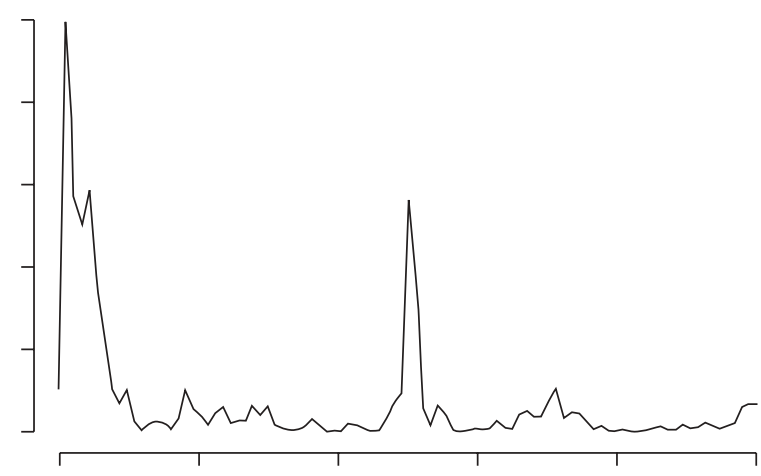

d)

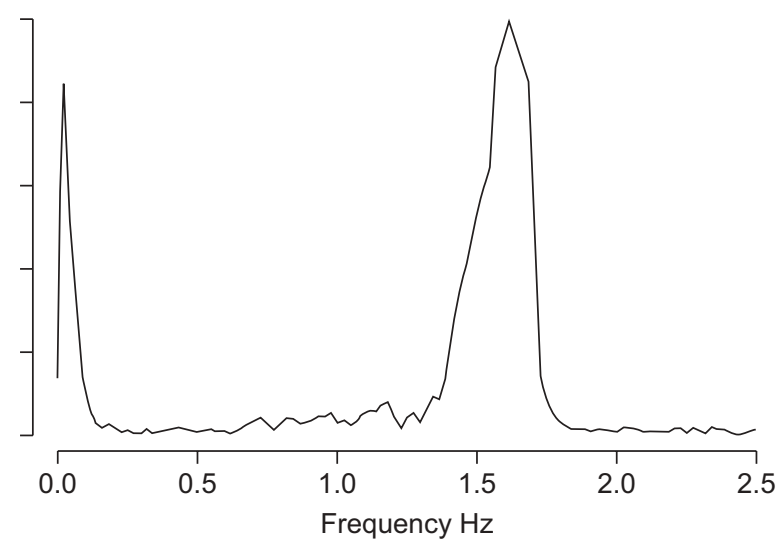

FIGURE 3. Representative traces of the power spectral density of heart rate variability in a) one sham-treated rat, b) one rat exposed to chronic intermittent hypoxia (CIH), c) one sham rat treated with ascorbic acid and d) one $\mathrm{CIH}$ rat treated with ascorbic acid. PSD: power spectral density expressed in normalised units (NU). 

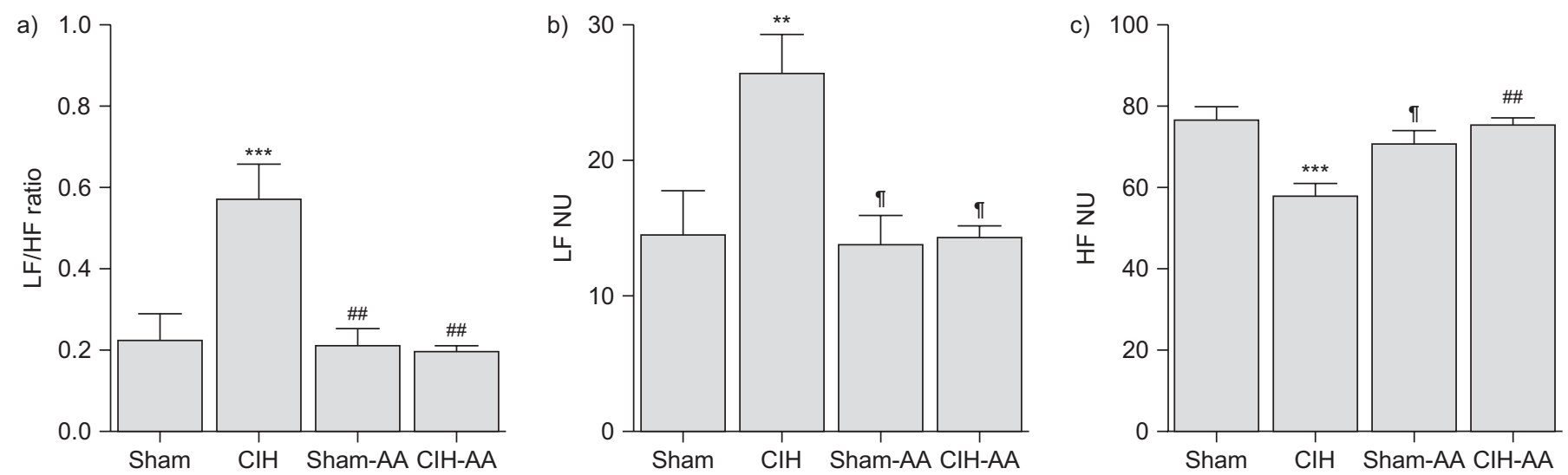

FIGURE 4. Spectral indexes of $R-R$ variability in sham rats $(n=10)$, chronic intermittent hypoxia $(C I H)$-exposed rats $(n=24)$, sham rats treated with ascorbic acid (sham$\mathrm{AA} ; \mathrm{n}=11)$ and $\mathrm{CIH}$ rats treated with ascorbic acid (CIH-AA; $n=10)$. a) Low-frequency (LF)/high-frequency (HF) ratio of power spectral density, b) $L F$ band expressed in normalised units $(\mathrm{NU})$ and $\mathrm{c}) \mathrm{HF}$ band expressed in $\mathrm{NU}$. ***: $p \leqslant 0.001$, and ${ }^{* *}: \mathrm{p} \leqslant 0.01$ compared to sham; ${ }^{* \#}: \mathrm{p} \leqslant 0.01$, and ${ }^{\circ}: \mathrm{p} \leqslant 0.05$ compared to $\mathrm{ClH}$.

known whether $\mathrm{CIH}$ increases ROS levels in $\mathrm{CB}$ chemoreceptor cells or in endothelial cells. PENG and co-workers [12, 15] proposed that ROS may enhance $\mathrm{CB}$ chemosensitivity by modifying the $\mathrm{O}_{2}$-sensitive $\mathrm{K}^{+}$channels in chemoreceptor cells, producing a large membrane depolarisation, which in turn increases the intracellular $\mathrm{Ca}^{2+}$ and the release of excitatory transmitters [24]. However, a direct excitatory effect of ROS on $\mathrm{CB}$ chemoreception is matter of debate [25]. An alternative
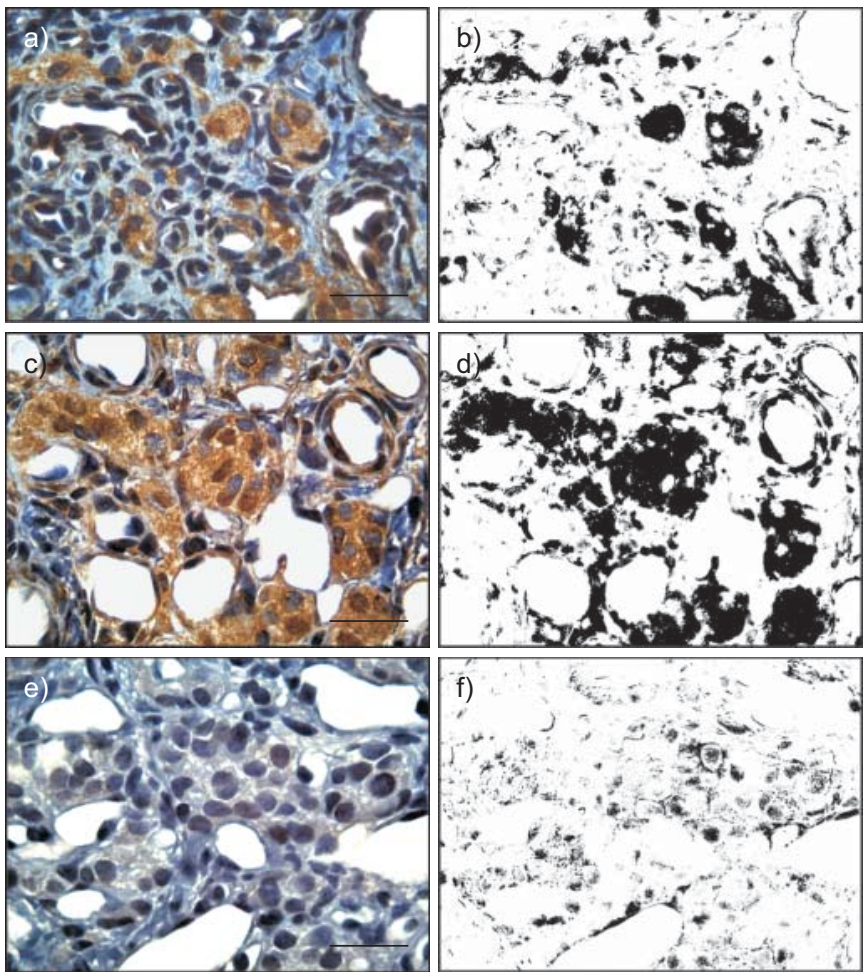

FIGURE 5. Micrographs showing positive immunoreactivity for 3-nitrotyrosine (3-NT) in carotid bodies from sham-treated rats ( $a$ and b), chronic intermittent hypoxia $(\mathrm{ClH})$-exposed rats $(\mathrm{c}$ and $\mathrm{d})$ and $\mathrm{Cl}$ rats treated with ascorbic acid (e and f). a, c and e) Immunohistochemistry. b, d and f) Images after colour deconvolution for quantification of 3-NT immunoreactivity. Scale bars $=20 \mu \mathrm{m}$. explanation is that an excessive ROS level may impair the mitochondrial metabolism, similarly to what is observed when the CB is stimulated with large amounts of NO [26]. Present results showing a marked increase of iNOS immunoreactivity and 3-NT immunoreactivity in the $\mathrm{CB}$ tissue suggest that $\mathrm{CIH}$ may increase NO production. It is known that iNOS is responsible for the overproduction of $\mathrm{NO}$ in several tissues, including the $\mathrm{CB}$ of rats exposed to chronic sustained hypoxia [27].
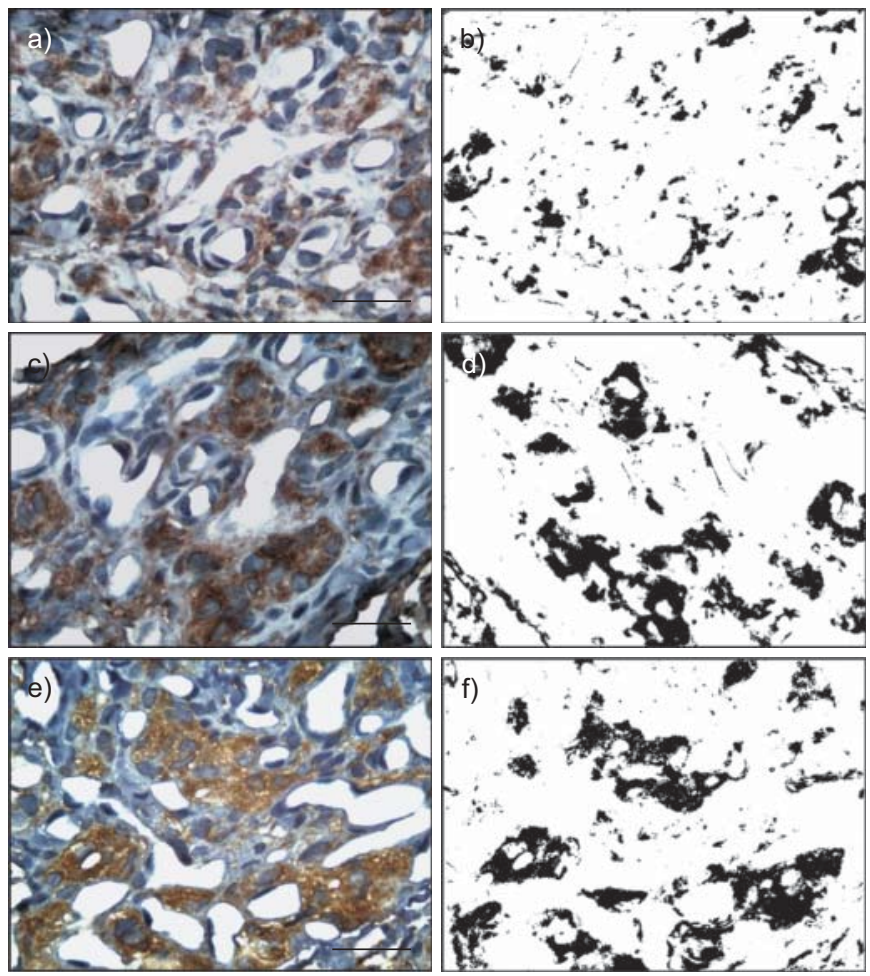

FIGURE 6. Micrographs showing positive immunoreactivity for inducible nitric oxide synthase (iNOS) in carotid bodies from sham-treated rats (a and b), chronic intermittent hypoxia $(\mathrm{ClH})$-exposed rats ( $\mathrm{c}$ and $\mathrm{d}$ ) and $\mathrm{ClH}$ rats treated with ascorbic acid (e and f). a, c and e) Immunohistochemistry. b, d and f) Images after colour deconvolution for quantification of iNOS immunoreactivity. Scale bars $=20 \mu \mathrm{m}$. 

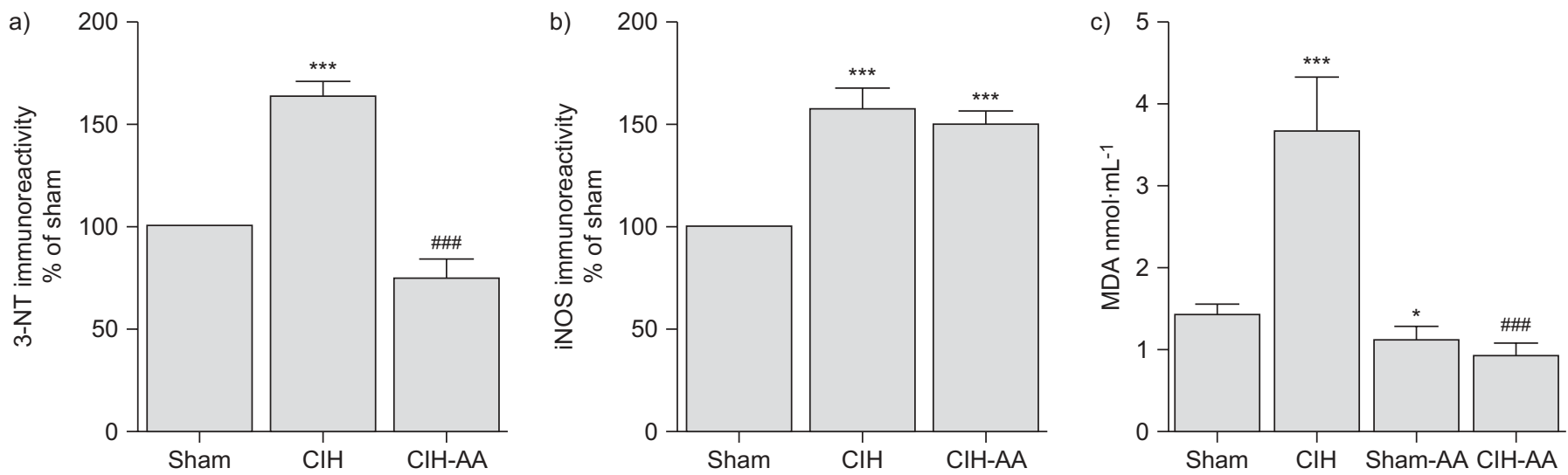

FIGURE 7. Summary of the effects of ascorbic acid on carotid body (CB) and systemic oxidative stress induced by chronic intermittent hypoxia (CIH). a) 3-Nitrotyrosine (3-NT) immunoreactivity and b) inducible nitric oxide synthase (iNOS) immunoreactivity measured in CBs from six sham-treated rats, eight rats exposed to $\mathrm{ClH}$ and five rats exposed to $\mathrm{ClH}$ and treated with ascorbic acid (CIH-AA). Data are presented as a percentage of sham immunoreactivity. c) Malondialdehyde (MDA) plasma levels in 14 sham, $10 \mathrm{ClH}$ and six $\mathrm{ClH}-\mathrm{AA}$ rats. ${ }^{* * *}: \mathrm{p} \leqslant 0.001$ versus sham; ${ }^{\# \# \#}: \mathrm{p} \leqslant 0.001$ versus $\mathrm{ClH} ;{ }^{*}: p \leqslant 0.05$ versus $\mathrm{ClH}$.

NO reacts with superoxide radical to form peroxynitrite, which undergoes heterolytic cleavage to form hydroxyl anion and nitronium ion, the latter of which nitrates protein tyrosine residues. The treatment with ascorbic acid prevented the increase of 3-NT immunoreactivity in the CB of CIH-rats, but did not reduce the increased iNOS immunoreactivity, suggesting that changes in iNOS expression are not directly dependent on the $\mathrm{CIH}$-induced oxidative stress. Since the hypoxic inducible factor $1 \alpha(\mathrm{HIF}-1 \alpha)$ plays a key role in the $\mathrm{CB}$ potentiation induced by $\mathrm{CIH}$ [28], it is likely that the high iNOS immunoreactivity found in $\mathrm{CBs}$ from $\mathrm{CIH}$ and $\mathrm{CIH}-\mathrm{AA}$ rats, could be the result of a direct activation of the HIF- $1 \alpha$ transcription pathway by the cyclic hypoxic pattern rather than by the oxidative stress. Our results suggest that iNOS plays a role in the mechanisms mediating the nitrotyrosine residue formation in the $\mathrm{CB}$, which in turn may lead to excessive NO production and functional losses associated with the chemosensory potentiation to hypoxia [26]. Li et al. [29] found that both iNOS expression and activity were upregulated by $\mathrm{CIH}$ hypoxia in mice, leading to significant peroxynitrite formation, neuronal injury and spatial memory deficit. In addition, ZHAN et al. [30] reported that transgenic absence of iNOS or pharmacological inhibition in mice confers resistance to $\mathrm{CIH}$-induced hypersomnolence and sleepiness, and protection against brain lipid peroxidation and proinflammatory gene overexpression. Thus, the available data support a critical role for iNOS in the development of the $\mathrm{CIH}$-induced oxidative stress and inflammatory responses, suggesting a potential role for inducible NO in OSA.

Few experimental studies have addressed the contribution of oxidative stress on the $\mathrm{CIH}$-induced hypertension. The finding that ascorbic acid prevents the hypertension and HRV alterations in CIH-rats confirm and extend previous observations showing that daily intraperitoneal administration of MnTMPyP abolished the hypertension and the increased plasma catecholamines in rats exposed to $\mathrm{CIH}$ [17]. In addition, our results showing that ascorbic acid prevents the potentiation of the ventilatory responses agree with the observation of MacFarlane and Mitchell [13], who found that MnTMPyP application into the intrathecal space of the cervical spinal cord abolished the phrenic long-term potentiation induced by acute intermittent hypoxia in rats. Thus, ROS formation seems to be needed for $\mathrm{CB}$ and motor respiratory plasticity induced by $\mathrm{CIH}$.

Our results support a preventing role for ascorbic acid supplementation on the oxidative stress, the potentiation of $\mathrm{CB}$ chemoreflex as well as on the development of HRV alterations and hypertension induced by $\mathrm{CIH}$. Thus, a potential therapeutic effect of antioxidants in OSA deserves further attention. However, a relevant concern is the human equivalence of the ascorbic acid dose used in this study. This is a crucial question that needs to be addressed before any clinical study can be carried out in humans. Scaling doses from animal studies to humans is a complex topic. The animal effective dose should not be extrapolated to the human equivalent dose (HED) by a simple conversion based on body weight. Contrarily, a normalisation based on body surface area (BSA) seems to be more appropriate [31]. If we assume that a $200 \mathrm{~g}$ rat drinks $\sim 10 \mathrm{~mL}$ of water per day containing $1.25 \mathrm{~g} \cdot \mathrm{L}^{-1}$ ascorbic acid, the daily consumed amount is about $62 \mathrm{mg} \cdot \mathrm{kg}^{-1}$. The HED formula for the dose translation based on BSA is calculated by multiplying the animal dose for the ratio of animal to human weight/BSA scaling factors [31]. Accordingly, if we translate the animal dose used here to the HED using the published scaling factors of 6 and 37, respectively for a $150 \mathrm{~g}$ rat and a $60 \mathrm{~kg}$ human, the HED dose is $10 \mathrm{mg} \cdot \mathrm{kg}^{-1}$, corresponding to a daily total of $600 \mathrm{mg}$ ascorbic acid. The only data published in humans are those by GREBE et al. [32], who demonstrated evidence of oxidative stress in OSA patients and improved flow-mediated vasodilatation in the brachial artery after acute infusion of $500 \mathrm{mg}$ ascorbic acid. Thus, the positive effects of antioxidants in OSA may extend beyond the effects on the CB.

In summary, present results show a preventive role for ascorbic acid in the generation of oxidative stress and the potentiation of $\mathrm{CB}$ chemoreflexes, as well as on the development of HRV and $\mathrm{BP}$ alterations induced by $\mathrm{CIH}$, supporting an essential role for systemic and local CB oxidative stress on the progression of the cardiorespiratory alterations induced by $\mathrm{CIH}$. 


\section{SUPPORT STATEMENT}

This work was supported by the National Fund for Scientific and Technological Development of Chile, grant FONDECYT 1070584.

\section{STATEMENT OF INTEREST}

None declared.

\section{REFERENCES}

1 McNicholas WT, Bonsignore MR, the Management Committee of ECAB. Sleep apnoea as an independent risk factor for cardiovascular disease: current evidence, basic mechanisms and research priorities. Eur Respir J 2007; 29: 156-178.

2 Somers VK, White DP, Amin R, et al. Sleep apnea and cardiovascular disease. J Am Coll Cardiol 2008; 52: 686-717.

3 Gozal D, Kheirandish-Gozal L. Cardiovascular morbidity in obstructive sleep apnea, oxidative stress, inflammation, and much more. Am J Respir Crit Care Med 2008; 177: 369-375.

4 Lavie L, Lavie P. Molecular mechanisms of cardiovascular disease in OSAHS: the oxidative stress link. Eur Respir J 2009; 33: 1467-1484.

5 Fletcher EC, Lesske J, Behm R, et al. Carotid chemoreceptors, systemic blood pressure, and chronic episodic hypoxia mimicking sleep apnea. J Appl Physiol 1992; 72: 1978-1984.

6 Narkiewicz K, Montano N, Cogliati C, et al. Altered cardiovascular variability in obstructive sleep apnea. Circulation 1998; 98: 1071-1077.

7 Iturriaga R, Moya EA, Del Rio R. Carotid body potentiation induced by intermittent hypoxia: implications for cardiorespiratory changes induced by sleep apnoea. Clin Exp Pharmacol Physiol 2009; 36: 1197-1204.

8 Prabhakar NR, Peng YJ, Jacono FJ, et al. Cardiovascular alterations by chronic intermittent hypoxia: importance of carotid body chemoreflexes. Clin Exp Pharmacol Physiol 2005; 32: 447-449.

9 Narkiewicz K, Van De Borne PJ, Pesek CA, et al. Selective potentiation of peripheral chemoreflex sensitivity in obstructive sleep apnea. Circulation 1999; 99: 1183-1189.

10 Peng YJ, Overholt JL, Kline D, et al. Induction of sensory long-term facilitation in the carotid body by intermittent hypoxia: implications for recurrent apneas. Proc Natl Acad Sci USA 2003; 100: 10073-10078.

11 Rey S, Del Rio R, Alcayaga J, et al. Chronic intermittent hypoxia enhances cat chemosensory and ventilatory responses to hypoxia. J Physiol 2004; 560: 577-586.

12 Peng YJ, Prabhakar NR. Reactive oxygen species in the plasticity of respiratory behavior elicited by chronic intermittent hypoxia. J Appl Physiol 2003; 94: 2342-2349.

13 MacFarlane PM, Mitchell GS. Respiratory long-term facilitation following intermittent hypoxia requires reactive oxygen species formation. Neuroscience 2008; 152: 189-197.

14 Rey S, Del Rio R, Iturriaga R. Contribution of endothelin-1 to the enhanced carotid body chemoreception induced by intermittent hypoxia. Brain Res 2006; 1086: 152-159.

15 Peng YJ, Nanduri J, Yuan G, et al. NADPH oxidase is required for the sensory plasticity of the carotid body by chronic intermittent hypoxia. J Neurosci 2009; 15: 4903-4910.
16 Chen L, Einbinder E, Zhang Q, et al. Oxidative stress and left ventricular function with chronic intermittent hypoxia in rats. $A m$ J Respir Crit Care Med 2005; 172: 915-920.

17 Kumar GK, Rai V, Sharma SD, et al. Chronic intermittent hypoxia induces hypoxia-evoked catecholamine efflux in adult rat adrenal medulla via oxidative stress. J Physiol 2006; 575: 229-239.

18 Lai CJ, Yang CCH, Hsu YY, et al. Enhanced sympathetic outflow and decreased baroreflex sensitivity are associated with intermittent hypoxia-induced systemic hypertension in conscious rats. J Appl Physiol 2006; 100: 1974-1982.

19 Reeves SR, Gozal E, Guo SZ, et al. Effect of long-term intermittent and sustained hypoxia on hypoxic ventilatory and metabolic responses in the adult rat. J Appl Physiol 2003; 95: 1767-1774.

20 Shiomi T, Guilleminault C, Sasanabe R, et al. Augmented very low frequency component of heart rate variability during obstructive sleep apnea. Sleep 1996; 19: 370-377.

21 Task Force of the European Society of Cardiology and the North American Society of Pacing and Electrophysiology. Heart rate variability. Standards of measurement, physiological interpretation, and clinical use. Eur Heart J 1996; 17: 354-381.

22 Rey S, Tarvainen MP, Karjalainen PA, et al. Dynamic time-varying analysis of heart rate and blood pressure variability in cats exposed to short-term chronic intermittent hypoxia. Am J Physiol Regul Integr Comp Physiol 2008; 295: R28-R37.

23 Shimokawa A, Kunitake T, Takasaki M, et al. Differential effects of anesthetics on sympathetic nerve activity and arterial baroreceptor reflex in chronically instrumented rats. J Auton Nerv Syst 1998; 72: 46-54.

24 Iturriaga R, Alcayaga J. Neurotransmission in the carotid body: transmitters and modulators between glomus cells and petrosal ganglion nerve terminals. Brain Res Rev 2004; 47: 46-53.

25 Gonzalez C, Agapito MT, Rocher A, et al. Chemoreception in the context of the general biology of ROS. Respir Physiol Neurobiol 2007; 157: 30-44.

26 Mosqueira M, Iturriaga R. Carotid body chemosensory excitation induced by nitric oxide: involvement of oxidative metabolism. Respir Physiol Neurobiol 2002; 131: 175-87.

27 Ye JS, Tipoe GL, Fung PCW, et al. Augmentation of hypoxiainduced nitric oxide generation in the rat carotid body adapted to chronic hypoxia: an involvement of constitutive and inducible nitric oxide synthases. Pflügers Arch 2002; 444: 178-185.

28 Peng YJ, Yuan G, Ramakrishnan D, et al. Heterozygous HIF-1 $\alpha$ deficiency impairs carotid body-mediated systemic responses and reactive oxygen species generation in mice exposed to intermittent hypoxia. J Physiol 2006; 577: 705-716.

29 Li RC, Row BW, Kheirandish L, et al. Nitric oxide synthase and intermittent hypoxia-induced spatial learning deficits in the rat. Neurobiol Dis 2004; 17: 44-53.

30 Zhan G, Fenik P, Pratico D, et al. Inducible nitric oxide synthase in long-term intermittent hypoxia: hypersomnolence and brain injury. Am J Respir Crit Care Med 2005; 171: 1414-1420.

31 Reagan-Shaw S, Nihal M, Ahmad N. Dose translation from animal to human studies revisited. FASEB J 2008; 22: 659-661.

32 Grebe M, Eisele HJ, Weissmann N, et al. Antioxidant vitamin C improves endothelial function in obstructive sleep apnea. Am J Respir Crit Care Med 2006; 173: 897-890. 